\title{
Plano de Reorganização da Atenção à Hipertensão Arterial e ao Diabetes Mellitus
}

\author{
Brazillian National Strategy for the Reorganization of Care for Arterial \\ Hipertension and Diabetes Mellitus
}

Secretaria de Políticas Públicas/MS*

\section{INTRODUÇÃO}

O grande impacto da morbimortalidade cardiovascular na população brasileira, que tem o diabetes mellitus (DM) e a hipertensão arterial (HA) como importantes fatores de risco, trazem um desafio para o sistema público de saúde: a garantia de acompanhamento sistemático dos indivíduos identificados como portadores desses agravos, assim como o desenvolvimento de ações referentes à promoção da saúde e à prevenção de doenças crônicas não transmissíveis.

O Plano de Reorganização da Atenção à Hipertensão Arterial e ao Diabetes Mellitus, implementado pelo Ministério da Saúde (MS), tem por objetivo estabelecer as diretrizes e metas para essa reorganização no Sistema Único de Saúde (SUS), investindo na atualização dos profissionais da rede básica, oferecendo a garantia do diagnóstico e proporcionando a vinculação do paciente às unidades de saúde para tratamento e acompanhamento, promovendo a reestruturação e a ampliação do atendimento resolutivo e de qualidade para os portadores. $\mathrm{O}$ atendimento eficiente e eficaz no sentido de contribuir para a redução da morbimortalidade associada à HA e ao DM no país depende, sobretudo, do estabelecimento de bases construídas a partir da pactuação solidária entre a União, estados e municípios, contando com o apoio e a participação das sociedades científicas e das entidades de portadores dessas patologias.

As primeiras etapas da reorganização são descritas estabelecendo os compromissos institucionais para sua operacionalização em todos os municípios brasileiros. As etapas mobilizadoras são fundamentais para criar o vínculo entre os portadores desses agravos e as equipes de atenção básica. Esse nível do SUS tem a capacidade de tratar e acompanhar mais de $65 \%$ dos casos detectados.

A Secretaria de Políticas de Saúde, do Ministério da Saúde, é responsável pela coordenação e gerência de todas as ações de implantação do Plano desenvolvidas em nível nacional, além da assessoria contínua aos estados e municípios.

\section{Plano de Reorganização da Atenção à Hipertensão Arterial e ao Diabetes Mellitus}

Apesar da predominância das doenças cardiovasculares como principal causa de mortalidade no Brasil, correspondendo a $27 \%$ - o equivalente a 255.585 mortes - do total de óbitos registrados no ano de 2000 (Sistema de Informação sobre Mortalidade SIM/MS), alguns fatores impediam que esse grupo de doenças tivesse, até então, obtido destaque na agenda dos gestores públicos de saúde.

Sendo a hipertensão arterial e o diabetes mellitus importantes fatores de risco para a morbimortalidade cardiovascular, o Ministério da Saúde implantou, no ano 2000, o Plano de Reorganização da Atenção à Hipertensão Arterial e ao Diabetes Mellitus no Brasil, contando com a parceria das Sociedades Brasileiras de Cardiologia, de Nefrologia, de Hipertensão e de Diabetes, Secretarias Estaduais e Municipais de Saúde, Conselhos Nacionais de Secretários Estaduais e de Secretários Municipais de Saúde, Federação Nacional de Portadores de Hipertensão e de Diabetes.

Essa grande estratégia, que visa a redução da morbimortalidade cardiovascular e a melhoria da qualidade de vida da população, prevê quatro etapas:

\section{Capacitação de multiplicadores para atualização} de profissionais da rede básica na atenção à HA e ao DM

Para operacionalizar essa atualização, o MS, em parceria com as secretarias estaduais e municipais de saúde e as sociedades científicas, iniciou a capacitação de multiplicadores dos estados e dos municípios. 
O segundo momento dessa etapa consiste em desenvolver uma estratégia de ação dirigida aos serviços de saúde da rede básica dos grandes centros urbanos, por meio da realização de uma proposta de educação permanente para os profissionais das unidades básicas de saúde dessas localidades.

O processo de educação permanente será auto-sustentável e se desenvolverá por meio de uma estratégia de capacitação, presencial e à distância, de médicos e enfermeiros, sob a coordenação da Secretaria de Políticas de Saúde/MS e responsabilidade das sociedades científicas parceiras.

\section{Campanha de informação e de identificação de casos suspeitos de HA e DM e Promoção de hábitos saudáveis de vida}

A identificação de suspeitos de DM pela rede básica do SUS na população com idade igual ou superior a 40 anos foi efetivada por campanha nacional, ocorrida no período de 6 de março a 7 de abril de 2001, constituindo-se o passo inicial para o processo de Reorganização da Atenção à Hipertensão Arterial e ao Diabetes Mellitus. Os indivíduos que apresentaram níveis alterados de glicemia também tiveram sua pressão arterial aferida, tendo em vista que a coexistência das duas situações aumenta o risco de complicações cardiovasculares.

Para que a população pudesse dispor de mais esclarecimentos sobre os fatores de risco e possíveis complicações decorrentes da HA e do DM, foram distribuídos materiais informativo e educativo sobre o assunto.

Ainda em 2001, em operação semelhante - também sob a forma de campanha nacional - serão identificados os casos suspeitos de HA. Essa etapa está prevista para realizar-se de 5 de novembro a 14 de dezembro de 2001.

Durante a campanha, será aferida a pressão arterial de todos aqueles que participarem da iniciativa e, em seguida, mediante a confirmação de casos suspeitos, os portadores de HA serão orientados pelos profissionais de saúde a estabelecer o vínculo com a rede básica do SUS.

\section{Confirmação diagnóstica e início da terapêutica}

Os casos suspeitos identificados de DM e HA devem ser vinculados às unidades básicas de saúde ou às equipes do Programa Saúde da Família, pelos quais serão solicitados os exames necessários para confirmação diagnóstica e início do tratamento, quando necessário.
A adesão ao tratamento é, seguramente, o maior desafio a ser enfrentado pelo plano, uma vez que se está lidando com doenças crônicas, pouco sintomáticas e cujo tratamento implica mudanças nos hábitos de vida de uma população numerosa. No entanto, deve-se ter presente essas dificuldades no sentido de buscar formas adequadas e criativas de enfrentamento dessa situação.

\section{Cadastramento, vinculação e acompanhamento dos pacientes portadores de HA e DM às Unidades Básicas de Saúde}

Todos os casos confirmados serão cadastrados e vinculados às unidades básicas de saúde e às equipes do Programa Saúde da Família e terão acompanhamento sistemático, clínico e laboratorial. Aqueles que necessitarem de atendimento especializado ou hospitalar serão encaminhados para os demais níveis de complexidade do sistema de saúde no próprio território ou em municípios vizinhos, segundo a negociação explicitada na Programação Pactuada Integrada (PPI) de cada estado. Esse cadastro possibilitará ao gestor federal, estadual ou municipal o planejamento para o atendimento desse público.

\section{Operacionalização geral do plano}

A pactuação entre os gestores do SUS é uma das principais estratégias para a implementação do Plano Nacional de Reorganização da Atenção à Hipertensão Arterial e ao Diabetes Mellitus. Assim, em outubro de 2000, foi realizada em Brasília a reunião nacional com os coordenadores estaduais do plano, com o presidente do Conselho Nacional de Secretários Municipais de Saúde (Conasems) e com o presidente do Conselho Nacional de Secretários Estaduais de Saúde (Conass). O objetivo do evento foi apresentar as diretrizes dessa reorganização, a Campanha Nacional de Detecção de Casos Suspeitos de Diabetes Mellitus e, também, a proposta de capacitação de profissionais de saúde que deverão atuar como multiplicadores nos estados e municípios.

O pacto entre os três níveis de gestão deu-se na reunião da Comissão Tripartite - CIT (outubro/2000). A operacionalização de cada etapa do plano está sendo discutida e acordada com os coordenadores estaduais designados pelas secretarias estaduais de saúde e com a Comissão Intergestores Bipartite para a pactuação em cada estado.

Com o objetivo de planejar, coordenar e acompanhar o desenvolvimento do plano em cada estado, foram criados comitês estaduais - constituídos por representantes das várias instâncias das SES, da coordenadoria estadual de controle de HA e DM, da área 
técnica de assistência farmacêutica, de representantes do conselho estadual dos secretários Municipais de Saúde; das sociedades científicas, das associações de portadores de HA e de DM e por coordenadores dos Pólos de Formação, Capacitação e Educação Permanente em Saúde da Família - sob orientação das Secretarias Estaduais de Saúde.

Os comitês constituem-se em espaço privilegiado para o acompanhamento das experiências bem-sucedidas e para a ampliação de parcerias entre governo e sociedade civil, processo de fundamental importância para o êxito das ações de reorganização da atenção aos portadores dessas patologias.

Para o acompanhamento desses comitês, foi organizado um grupo de consultores, disponíveis para visitas periódicas aos estados. Esses profissionais desenvolvem atividades de supervisão, capacitação dos membros dos comitês estaduais e assessoria em cada estado no desenvolvimento das ações para operacionalização e implementação do plano.

O Ministério da Saúde produziu 45 mil exemplares do Caderno Técnico sobre Hipertensão e Diabetes para a Atenção Básica e 15 mil exemplares de Casos clínicos de HA e DM, para serem utilizados nos cursos de capacitação.

Para a implantação das normas estabelecidas nos materiais didáticos, serão oferecidos cursos de capacitação presencial, além de cursos de educação à distância sobre HA e DM, para os profissionais da atenção básica de nível superior, ação sob a responsabilidade das Sociedades Brasileiras de Cardiologia, de Nefrologia, de Hipertensão e de Diabetes.

A oferta de exames complementares, para identificar alterações metabólicas e lesões em órgãos-alvo, é de fundamental importância para o acompanhamento dos portadores de HA e DM. Para garantir a realização dos exames complementares e o acompanhamento dos casos que demandarem atenção especializada ou hospitalar, será necessário o estabelecimento de fluxo para referência e contra-referência. Esse tem sido um dos grandes desafios da organização da rede de serviços de saúde em todo o país. A NOAS-SUS 01/2001 representa uma possibilidade concreta de formalizar os fluxos de referência e contra-referência entre gestores municipais com a presença efetiva do gestor estadual na regulação da oferta dos procedimentos destinados a controlar problemas prioritários como a HA e o DM, mediante negociação explicitada na Programação Pactuada Integrada.

Em 1998, depois de amplo debate para a sua elaboração, com a participação de toda a sociedade envolvi- da com o tema medicamentos, o Ministério da Saúde aprovou a Política Nacional de Medicamentos (PNM). Essa política fortalece os princípios e as diretrizes constitucionais e legalmente estabelecidos, explicitando, além das diretrizes básicas, as prioridades a ser conferidas em sua implementação e as responsabilidades dos gestores do SUS em sua efetivação.

Parte essencial da Política Nacional de Saúde, a PNM do Brasil, aprovada com a edição da Portaria $\mathrm{GM} \mathrm{N}^{\circ} 3.916$, de 30/10/98, constitui um dos elementos fundamentais para a efetiva implementação de ações capazes de promover a melhoria das condições da assistência à saúde da população.

A PNM estabeleceu diretrizes norteadoras de todas as ações, nas três esferas de governo, aos gestores do SUS, atuando em estreita parceria, objetivando assegurar o acesso da população a medicamentos seguros, eficazes e de qualidade, ao menor custo possível.

Uma das diretrizes fundamentais da PNM é a reorientação da assistência farmacêutica. $\mathrm{O}$ modelo de assistência farmacêutica está sendo reorientado de modo a que não se restrinja à aquisição e à distribuição de medicamentos.

O processo de implementação da PNM vem exigindo a definição de planos, programas e atividades específicas nas esferas federal, estadual e municipal. Como exemplos, no nível federal, a aprovação da Relação Nacional de Medicamentos (Rename), após 16 anos, com a edição da Portaria GM N ${ }^{\circ}$ 507/99, de 19/5/99. Nas esferas estadual e municipal, a implementação e a operacionalização da Portaria GM N ${ }^{\circ}$ 176/99, de 8/3/99, que instituiu o incentivo à Assistência Farmacêutica Básica, numa nova lógica de gestão, num novo modelo descentralizado.

O plano, em si, contempla o estabelecimento de diretrizes voltadas ao processo de detecção e tratamento dos portadores de HA e DM, no âmbito do Sistema Único de Saúde (SUS). Já foram definidos três medicamentos a ser distribuídos gratuitamente na rede pública de saúde para o tratamento de pessoas identificadas como portadoras de hipertensão: captopril $25 \mathrm{mg}$, hidroclorotiazida $25 \mathrm{mg}$ e propranolol $40 \mathrm{mg}$. O MS estima um investimento de R\$ 140 milhões para a compra de 7,8 milhões de comprimidos. Essa quantidade garante o tratamento de 11,1 milhões de pacientes, durante 12 meses.

Quanto ao tratamento dos diabéticos, definiu-se pela utilização, além da insulina NPH-100, disponibilizada na rede sistematicamente, dos hipoglicemiantes orais glibenclamida $5 \mathrm{mg}$ e da metformina $500 \mathrm{mg}$ e $850 \mathrm{mg}$. 
A glibenclamida já está sendo distribuída a todos os municípios do país, num total de 172 milhões de comprimidos, representando uma cobertura de mais de 1,7 milhão de portadores de diabetes em uso de medicação por período de seis meses. Encontra-se em fase de aquisição o medicamento metformina para que seja, também, distribuído a todos os municípios brasileiros.

O Ministério da Saúde, por meio da Secretaria de Políticas de Saúde, em parceria com o Conass e Conasems, traçou estratégias complementares no tocante ao processo de aquisição e disponibilização desses medicamentos.

Vale ressaltar que os medicamentos incluídos nessas ações de operacionalização do plano são medicamentos considerados "essenciais" pela OMS e fazem parte da atual Relação Nacional de Medicamentos Essenciais (Rename) do Brasil.

\section{Promoção da saúde e prevenção das doenças crônicas não transmissíveis}

É necessário que estados e municípios fortaleçam suas ações de promoção da saúde e prevenção de doenças não transmissíveis, pela implantação e implementação de programas como o Projeto Carmen (Conjunto de Ações para Redução Multifatorial das Enfermidades Não-Transmissíveis) e as ações para a introdução de práticas regulares de atividade física junto à população.

$\mathrm{O}$ estímulo à prática regular da atividade física, como proposto no Programa do MS Agita Brasil, é uma estratégia de melhoria das condições de vida e saúde da população no sentido de promover a qualidade de vida. Esse programa, baseado na experiência do município de São Paulo - o Agita São Paulo - tem o propósito de reduzir o sedentarismo, importante fator de risco para as doenças cardiovasculares.

O Projeto Carmen é uma iniciativa da Organização Mundial da Saúde (OMS) e da Organização Pan-Americana da Saúde (OPS) e tem por objetivo a redução e o controle dos fatores de risco das doenças não-transmissíveis e já está implantado na região leste da cidade de Goiânia, no Estado de Goiás.

\section{CONCLUSÕES}

Atualmente, dentre as causas mais importantes de internações hospitalares bem como de mortalidade, destacam-se a hipertensão arterial e o diabetes mellitus. A operacionalização do Plano de Reorganização da Atenção à Hipertensão Arterial e ao Diabetes Mellitus, respaldado pela comunidade científica brasileira, poderá contribuir para mudar radicalmente o curso até então conhecido dessas doenças e de suas complicações.

Conforme estabelecido, o plano tem na educação e promoção à saúde seus alicerces, mas não pode prescindir das evidências acumuladas de prevenção secundária contra as complicações, as quais necessariamente incluem tratamento farmacológico e exames a ser oferecidos ainda na atenção básica.

O sucesso do plano, num país com as dimensões e heterogeneidades regionais observadas no Brasil, está diretamente relacionado à adesão de todas as entidades governamentais e não-governamentais envolvidas com esses importantes problemas de saúde pública.

Assim, é fundamental a construção de um consenso de natureza político-administrativa e técnico-científica que sustente o trabalho em parceria entre os gestores do SUS, sociedades científicas e associações de portadores de HA e de DM, identificando responsabilidades e necessidades de apoio mútuo. 